\title{
Peritherapeutic Hemodynamic Changes of Carotid Stenting Evaluated with Quantitative DSA in Patients with Carotid Stenosis
}

\author{
(D) M.M.H. Teng, (DF.-C. Chang, (D) C.-J. Lin, (D) Chiang, (D).-S. Hong, and (D)Y.-H. Kao
}

\begin{abstract}
BACKGROUND AND PURPOSE: Quantitative data from DSA have become important tools for understanding hemodynamic changes of intracranial lesions. In this study, we evaluated 8 hemodynamic parameters in patients before and after carotid artery angioplasty.
\end{abstract}

MATERIALS AND METHODS: DSA images of 34 patients with carotid stenosis who underwent angioplasty and stent placement were retrospectively analyzed. Eleven ROls ( $M 1, M 2$, Al, A2, the parietal vein, superior sagittal sinus, internal jugular vein, and 4 in the ICA) were selected on color-coded DSA. Eight hemodynamic parameters (bolus arrival time, TTP, relative TTP, full width at half maximum, wash-in slope, washout slope, maximum enhancement, and area under the curve) were measured from the time-concentration curves of these ROls. The dependent $t$ test for paired samples was applied to these parameters before and after stent placement.

RESULTS: We found that the treatment significantly reduced TTP, relative TTP, bolus arrival time, and washout slope at all arterial ROIs and full width at half maximum and area under the curve at some arterial ROIs. Bolus arrival time was significantly reduced after treatment for all arterial ROIs, the parietal vein, and the superior sagittal sinus. The maximum enhancement and wash-in slope did not show significant changes after treatment. After treatment, the relative TTP from the ICA to M1, M2, and the parietal vein returned to normal values.

CONCLUSIONS: In addition to TTP and relative TTP, other parameters can be used to evaluate peritherapeutic cerebral hemodynamic changes. Bolus arrival time has the potential to evaluate brain circulation at arterial and venous sites, especially when TTP cannot be measured because of an incomplete time-concentration curve.

ABBREVIATIONS: $A \cup C=$ area under the curve; $B A T=$ bolus arrival time; $F W H M=$ full width at half maximum; $\mathrm{ME}=$ maximal enhancement; $\mathrm{PA}=$ posterioranterior; $\mathrm{PV}$ = parietal vein; $\mathrm{rTTP}=$ relative TTP; SSS = superior sagittal sinus; $\mathrm{WI}=$ wash-in slope; $\mathrm{WO}=$ washout slope

D igital subtraction angiography has been the criterion standard for diagnosing cerebral vascular disease since 1970s. DSA imaging demonstrates the intravascular changes of concentration in the time domain and can be used as a surrogate marker for cerebral hemodynamics. Time-concentration curve analysis is the most commonly used approach because the results are easily

Received December 16, 2015; accepted after revision March 20, 2016.

From the Department of Medical Imaging (M.M.H.T.), Cheng Hsin General Hospital, Taipei, Taiwan; Department of Radiology (F.-C.C., C.-J.L., L.C.), Taipei Veterans General Hospital, Taipei, Taiwan; and School of Medicine (F.-C.C., C.-J.L.) and Department of Biomedical Imaging and Radiological Sciences (J.-S.H., Y.-H.K.), National Yang-Ming University, Taipei, Taiwan.

This work was supported, in part, by the Cheng Hsin General Hospital and National Yang Ming University cooperative project (grant No. 103F003C10) and Taipei Veterans General Hospital (grant No. V104-C-012).

Please address correspondence to Chung-Jung Lin, PhD, Radiology Department, Taipei Veterans General Hospital, No. 201, Section 2, Shipai Rd, Beitou District, Taipei, Taiwan; e-mail: bcjlin@gmail.com; Yi-Hsuan Kao, MD, Department of Biomedical Imaging and Radiological Sciences, National Yang-Ming University, No. 155, Section 2, Li-Nong St, Beitou District, Taipei, Taiwan; e-mail: yhkao@ym.edu.tw

http://dx.doi.org/10.3174/ajnr.A4813 accessible and intuitive for interventional purposes. Time-topeak (also called time-to-maximum in some previous publications) is the most commonly used parameter. It is applied to detect intra-aneurysmal flow, and larger aneurysms demonstrate more prolonged TTP than smaller aneurysms. ${ }^{1}$ The difference in TTP between the parietal vein and cavernous ICA has been used to monitor cerebral hemodynamic changes to improve patient safety. ${ }^{2}$ Detecting shorter TTPs in carotid cavernous fistulas helps determine whether the venous outlets contain higher blood flow, which deserves treatment priority. ${ }^{3}$ In control angiography, normalization of blood flow indicates spontaneous latent obliteration. ${ }^{4}$ Other applications include monitoring angioplasty for vasospasm after subarachnoid hemorrhage ${ }^{5}$ and quantitatively grading Moyamoya disease. ${ }^{2}$

The standard gray-scale images of DSA are first encoded into a single composite color image according to the contrast bolus arrival time (BAT) at each point in the circulatory system of the brain. This single composite color image improves the speed of manual delineation of AVMs and makes the angioarchitecture 

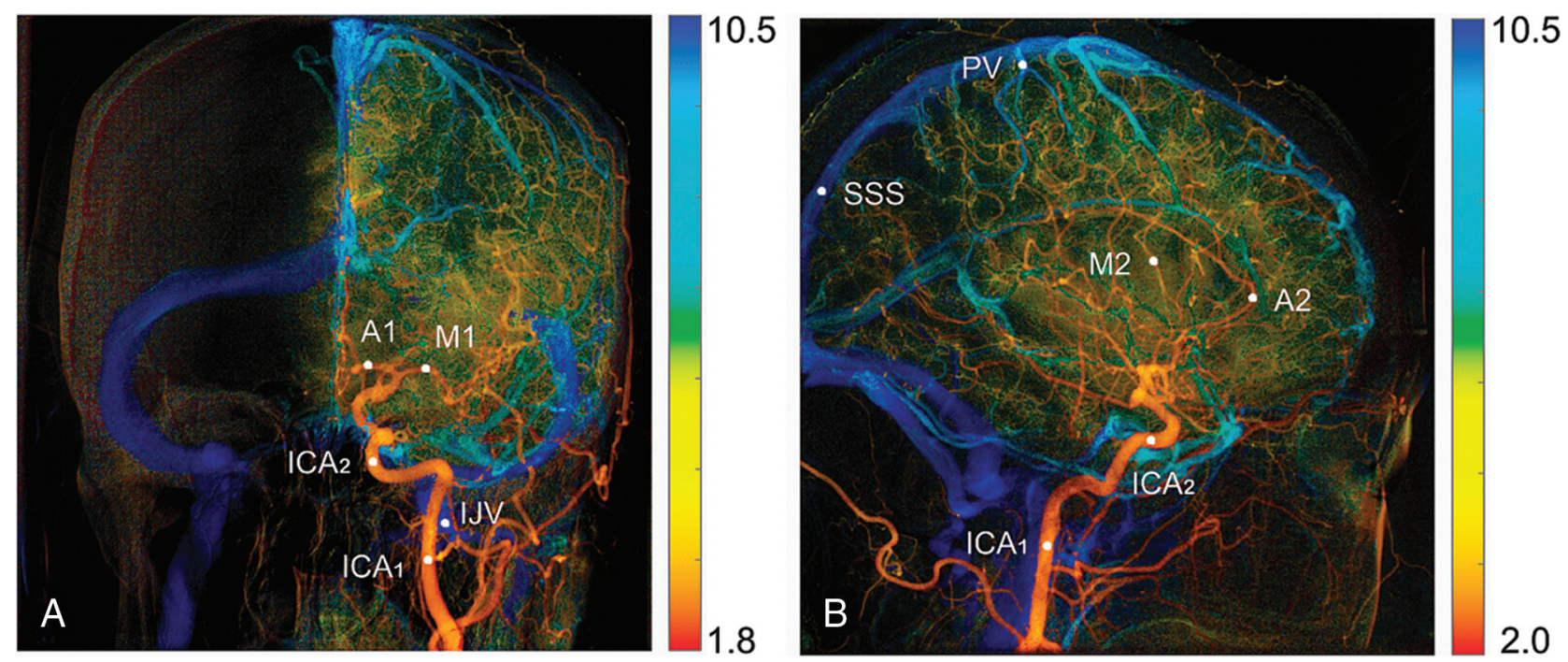

FIG 1. Color-coded DSA images in the PA $(A)$ and lateral $(B)$ views. Eleven ROIs are manually selected for quantification of perfusion parameters. $I C A_{1}$ on the PA view and ICA on lateral view are planned to be at the same place.
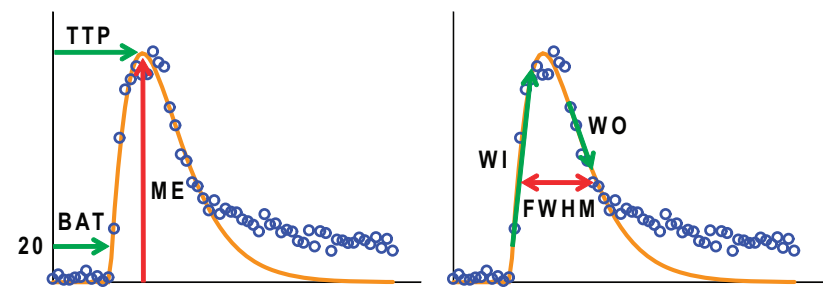

FIG 2. The measured (blue open circle) and fitted (orange line) timeconcentration curves. Hemodynamic parameters such as maximum enhancement, time to peak, bolus arrival time, wash-in slope, washout slope, and full width at half maximum are all derived from the fitted curve.

and the flow characteristics of aneurysms, AVMs, fistulas, stenosis, occlusions, dissections, and tumors easier to understand. ${ }^{6}$ The color-coding of DSA was also developed by calculation of the maximal enhancement (ME) and the TTP of the time-concentration curve of each pixel. ${ }^{7}$ It improves the conspicuity of findings on DSA images of cerebral vascular lesions and helps with the visualization of fistula angioarchitecture and venous drainage patterns in patients with carotid cavernous fistulas. ${ }^{7}$ In addition, it facilitates real-time hemodynamic monitoring, helps determine the end point of embolization, and increases patient safety in the treatment of carotid cavernous fistulas. ${ }^{8,9}$

For carotid stenosis, the TTP or relative TTP (rTTP) of colorcoded quantitative DSA has been used to evaluate cerebral circulation time and peritherapeutic hemodynamic changes in the intracranial circulation. ${ }^{3}$ rTTP was found to be a definitive alternative method of measuring cerebral circulation. ${ }^{10}$ Maximal slope and full width at half maximum (FWHM) are found to complement rTTP in the evaluation of cerebral circulation. ${ }^{10}$ Furthermore, the area under the curve (AUC) of the proximal common carotid arteries can be calculated from the time-concentration curves of color-coded DSA. A good correlation was found between the AUC and the relative CBV obtained from flat panel detector CT by injecting contrast medium into the ascending aorta. ${ }^{11}$ In this study, we evaluated 8 parameters obtained from color-coded quantitative DSA to understand the hemodynamic changes before and after stent placement in patients with carotid stenosis.

\section{MATERIALS AND METHODS}

DSA images of 34 patients (mean age, 72.3 years; 27 men and 7 women) who underwent carotid angioplasty and stent placement were collected for retrospective analysis. The patients had unilateral carotid stenosis of $>70 \%$, according to the NASCET criteria. The DSA images were acquired on a clinical scanner (Artis zee; Siemens, Erlangen, Germany) with a frame rate of 6 frames/s for $9 \sim 12$ seconds. The image size was $1440 \times 1440$ pixels, the FOV was $22 \mathrm{~cm}$, and the pixel size was $0.154 \times 0.154 \mathrm{~mm}^{2}$. A power injector (Liebel-Flarsheim Angiomat; Illumina, San Diego, California) was used to inject the contrast bolus in the common carotid artery at the $\mathrm{C} 4$ vertebral body level, by using a $4 \mathrm{~F}$ angiocatheter. A bolus of $12 \mathrm{~mL}$ of $60 \%$ diluted contrast medium $(340 \mathrm{mg} \mathrm{I} / \mathrm{mL}$ ) was administered in 1.5 seconds. The injection catheter was placed at the same place in the pre- and postintervention acquisitions. The injection was synchronized with the start of the image acquisition. The scanning protocol was the same in the 2 sets of images obtained before and after stent placement. This retrospective study was approved by the institutional review board.

Image postprocessing was performed on a personal computer by using software programs written in Matlab (MathWorks, Natick, Massachusetts). In our Matlab program, the TTP and ME values of the time-concentration curve for each pixel were used to generate color-coded DSA images (Fig 1). The first image obtained in each view of the angiograms was defined as time $=0$ for the TTP calculation. The color spectrum was used to represent TTP values, with a hot color (red) assigned to small TTP values and a cold color (blue) assigned to large TTP values. The ME was used to assign the brightness of these colors.

Eleven ROIs were selected for comparing hemodynamic parameters. The ROI was composed of $3 \times 3$ pixels with an area of $0.462 \times 0.462 \mathrm{~mm}^{2}$. On the posterior-anterior $(\mathrm{PA})$ view, we selected 5 ROIs: the internal carotid artery in 2 locations $\left(\mathrm{ICA}_{1}\right.$ and $\mathrm{ICA}_{2}$ ) and the $\mathrm{A} 1, \mathrm{M} 1$, and internal jugular vein. On the 

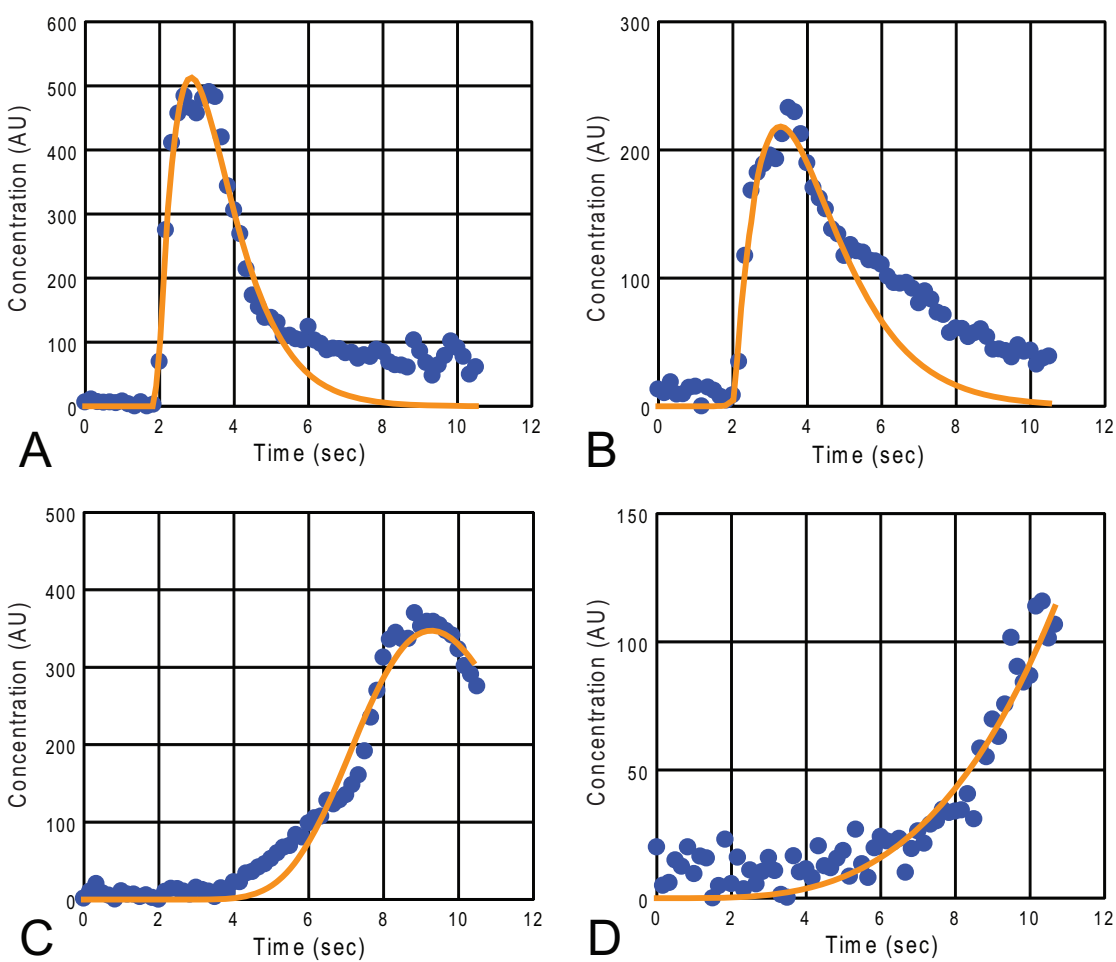

FIG 3. Measured (blue circle) and fitted (orange line) time-concentration curves of representative ROls before the stent-placement treatment: ICA $(A), M 2(B)$, PV (C), SSS (D). Note that the timeconcentration curve of the PV $(C)$ lacks the middle and lower descending portion. The ME in the time-concentration curve of the SSS $(D)$ is at the last temporal point. AU indicates arbitrary unit.

Table 1: $\boldsymbol{P}$ values of all hemodynamic parameters before and after stenting at each $\mathrm{ROI}^{\mathrm{a}}$

\begin{tabular}{|c|c|c|c|c|c|c|c|c|c|c|c|}
\hline \multirow{2}{*}{$\begin{array}{c}\text { ROI } \\
\text { Locations }\end{array}$} & \multicolumn{5}{|c|}{ PA View } & \multicolumn{6}{|c|}{ Lateral View } \\
\hline & $I \mathrm{CA}_{1}$ & $I C A_{2}$ & M1 & $\mathrm{A} 1$ & IJV & $I \mathrm{CA}_{1}$ & $I C A_{2}$ & $\mathrm{M} 2$ & $\mathrm{~A} 2$ & PV & SSS \\
\hline $\mathrm{ME}$ & .27 & .11 & .15 & .79 & NA & .68 & .99 & .20 & * & .55 & NA \\
\hline TTP & * & * & * & * & NA & * & * & * & * & * & NA \\
\hline rTTP & - & $\star b$ & $\star b$ & $\star b$ & NA & - & $\star c$ & $\star d$ & $\star d$ & $\star d$ & NA \\
\hline BAT & * & * & * & * & .32 & * & * & * & * & * & * \\
\hline FWHM & * & * & * & .08 & NA & * & * & * & * & NA & NA \\
\hline WI & .97 & .88 & .09 & .64 & .15 & .37 & .20 & .12 & .06 & .20 & NA \\
\hline WO & * & * & * & * & NA & * & * & * & * & NA & NA \\
\hline AUC & * & * & .06 & .39 & NA & * & * & * & .83 & NA & NA \\
\hline
\end{tabular}

Note:-NA indicates not available because of incomplete data; -, not calculated; *, significant difference before and after stenting $(P<.05)$; IJV, internal jugular vein.

${ }^{a}$ Details for significant results are shown in Tables 2 and 3.

b TTP relative to $I C A_{1}$ in the PA view.

'TTP relative to $I C A_{1}$ in the lateral view.

${ }^{\mathrm{d}}$ TTP relative to $I C A_{2}$ in the lateral view.

lateral view, we selected 6 ROIs: $\mathrm{ICA}_{1}, \mathrm{ICA}_{2}$ (at middle of cavernous segment of the ICA), the A2, M2, parietal vein (PV), and the superior sagittal sinus (SSS). The PA view of $\mathrm{ICA}_{1}$ and the lateral view of $\mathrm{ICA}_{1}$ were selected at the same place in the upper neck because they were easily and consistently identified in the respective views. The locations of these ROIs are shown in Fig 1.

The time-concentration curves of the selected ROIs were fitted to a gamma-variate function described by

$$
C_{\gamma}(t)=K\left(t-t_{0}\right)^{\alpha} \cdot e^{-\left(t-t_{0}\right) / \beta},
$$

where $K$ is a constant, $t_{0}$ is the contrast arrival time of the fitted curve, and $\alpha$ and $\beta$ are related to the wash-in and washout phases of the curve, respectively. ${ }^{12}$ The trust-region-reflective algorithm was used in the Matlab curve-fitting subroutine. ${ }^{13,14}$ An example is shown in Fig 2. The concentration is represented in arbitrary units.

According to the literature, the fitted $t_{0}$ does not reliably represent the BAT. ${ }^{15}$ We calculated the BAT by using the first time point with a concentration higher than 20 arbitrary units. To evaluate the wash-in and washout of the contrast media, we fitted 4 consecutive temporal data points of $C_{\gamma}(t)$ to a straight line described by

2)

$$
C_{\gamma}(t)=m t+b,
$$

by using a least squares technique. ${ }^{16}$ The largest and smallest m-values were recorded to represent the wash-in slope (WI) and washout slope (WO), respectively. The ME, TTP, full width at half maximum, and the area under the curve of the fitted curve were measured to depict the curve.

The hemodynamic parameters, ME, TTP, BAT, FWHM, WI, WO, and AUC, measured on the ROIs before and after the stent placement treatment, were evaluated. The dependent $t$ test for paired samples described by

$$
t=\frac{\overline{X_{\mathrm{M}}}-\mu_{0}}{\overline{X_{\mathrm{SD}}} / \sqrt{n}},
$$

was applied to the measured hemodynamic parameters before and after treatment, where $\overline{X_{\mathrm{M}}}$ and $\overline{X_{\mathrm{SD}}}$ were the mean and SD of the difference in a hemodynamic parameter measured before and after stent placement treatment, $\mu_{0}=0$, and $n$ is the number of measurements.

\section{RESULTS}

The anterior cerebral artery may not appear on the angiogram before stent placement because of insufficient blood flow. We measured the A1 in 18 cases and the A2 in 17 cases because their ROIs could be identified both before and after treatment on their color-coded images. All identified arterial ROIs, including the ICAs, M1, M2, A1, and A2, in these cases were successfully evaluated with all 8 parameters.

Figure 3 shows measured and fitted time-concentration curves of representative ROIs. Before stent placement, the MEs of the identified SSS in 16 patients and the internal jugular vein in 28 patients were found from the last image in their time-concentration curves (eg, Fig 3D). Assuming that the TTP occurred at the last time point was not correct. Therefore, TTP, rTTP, ME, FWHM, WO, and AUC of the SSS and internal jugular vein were listed as not measurable in Table 1. Furthermore, the FWHM, WO, or AUC was not measurable or not reliable for some ROIs in the PV and some other veins because of early termination in the washout phase (eg, Fig 3C). Only the BAT could be evaluated in all ROIs.

Figure 4 demonstrates the measured and fitted time-concen- 

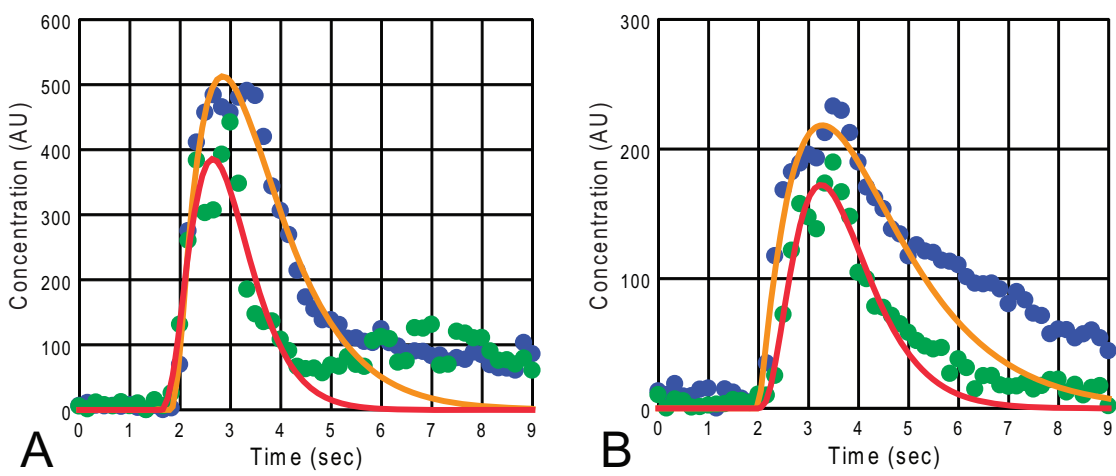

FIG 4. A comparison of the time-concentration curves before and after the stent placement treatment for the $\mathrm{ICA}_{2}(A)$ and $\mathrm{M} 2(B)$ in the lateral view. The blue solid circles are measured data, and orange lines are the fitted curves before the treatment. The green solid circles and red lines are measured data and fitted curves, respectively, after treatment. AU indicates arbitrary unit.

Table 2: Results for the hemodynamic parameters with significant changes $(P<.05)$ after the stenting procedure in the PA view

\begin{tabular}{|c|c|c|c|}
\hline ROI & $\begin{array}{c}\text { Before Treatment } \\
(\text { Mean } \pm \text { SD) }\end{array}$ & $\begin{array}{l}\text { After Treatment } \\
(\text { Mean } \pm \text { SD) }\end{array}$ & No. \\
\hline \multicolumn{4}{|l|}{ TTP (sec) } \\
\hline $\mathrm{ICA}_{1}$ & $2.89 \pm 0.62$ & $2.46 \pm 0.27$ & 34 \\
\hline $\mathrm{ICA}_{2}$ & $3.16 \pm 0.70$ & $2.60 \pm 0.25$ & 34 \\
\hline $\mathrm{Ml}$ & $3.70 \pm 0.92$ & $2.95 \pm 0.31$ & 34 \\
\hline $\mathrm{Al}$ & $3.42 \pm 0.67$ & $2.95 \pm 0.37$ & 18 \\
\hline \multicolumn{4}{|l|}{ rTTP (sec) } \\
\hline$I C A_{1}-I C A_{2}$ & $0.27 \pm 0.22$ & $0.14 \pm 0.13$ & 34 \\
\hline $\mathrm{ICA}_{1}-\mathrm{MI}$ & $0.81 \pm 0.43$ & $0.49 \pm 0.18$ & 34 \\
\hline$\left|C A_{1}-A\right|$ & $0.74 \pm 0.25$ & $0.54 \pm 0.23$ & 18 \\
\hline \multicolumn{4}{|l|}{ BAT (sec) } \\
\hline $\mathrm{ICA}_{1}$ & $1.37 \pm 0.29$ & $1.15 \pm 0.20$ & 34 \\
\hline $\mathrm{ICA}_{2}$ & $1.63 \pm 0.37$ & $1.27 \pm 0.29$ & 34 \\
\hline $\mathrm{Ml}$ & $1.91 \pm 0.48$ & $1.49 \pm 0.31$ & 34 \\
\hline $\mathrm{A} 1$ & $1.85 \pm 0.45$ & $1.51 \pm 0.31$ & 18 \\
\hline \multicolumn{4}{|l|}{ FWHM (sec) } \\
\hline $\mathrm{ICA}_{1}$ & $2.54 \pm 0.81$ & $1.95 \pm 0.46$ & 34 \\
\hline $\mathrm{ICA}_{2}$ & $2.53 \pm 0.89$ & $1.96 \pm 0.50$ & 34 \\
\hline $\mathrm{M} 1$ & $3.27 \pm 0.91$ & $2.62 \pm 0.63$ & 34 \\
\hline \multicolumn{4}{|l|}{$\mathrm{WO}(\mathrm{AU} / \mathrm{s})$} \\
\hline $\mathrm{ICA}_{1}$ & $-209 \pm 79$ & $-264 \pm 80$ & 34 \\
\hline $\mathrm{ICA}_{2}$ & $-202 \pm 80$ & $-247 \pm 91$ & 34 \\
\hline $\mathrm{Ml}$ & $-86 \pm 44$ & $-112 \pm 44$ & 34 \\
\hline $\mathrm{A} 1$ & $-73 \pm 29$ & $-90 \pm 30$ & 18 \\
\hline \multicolumn{4}{|l|}{$\mathrm{AUC}(\mathrm{AU} / \mathrm{sec})$} \\
\hline $\mathrm{ICA}_{1}$ & $1268 \pm 426$ & $942 \pm 324$ & 34 \\
\hline $\mathrm{ICA}_{2}$ & $1188 \pm 476$ & $866 \pm 269$ & 34 \\
\hline
\end{tabular}

Note:-No. indicates number of cases measured; AU, arbitrary unit.

tration curves for ROIs at the $\mathrm{ICA}_{2}$ and $\mathrm{M} 2$ in the lateral view, obtained before and after the stent-placement procedure. In this case, the contrast media arrived at these arteries at similar times, but they flushed out faster after the stent placement was performed. As a result of the faster washout of the contrast media, the FWHM and WO for these arteries decreased significantly after stent placement.

Table 1 is a list of comparisons of the hemodynamic parameters before and after stent placement at different ROIs. According to Table 1, TTP and BAT showed significant differences before and after stent placement in 9 and 10 ROIs, respectively. The TTP, BAT, and WO showed significant changes before and after stent placement at all arterial ROIs and FWHM for most arterial ROIs. Among the venous ROIs measured, significant changes in TTP were found at the PV, and significant changes in the BAT were found at the PV and SSS. The ME and WI had no value in demonstrating the hemodynamic changes before and after stent placement. The $P$ values of many of the venous ROIs were not available because their time-concentration curves did not reach a maximum or lacked a sufficient downward curve.

The statistical data for hemodynamic parameters with significant changes are shown in the PA (Table 2) and lateral (Table 3) views. For the TTP calculation, the first image was defined as time $=0$. The rTTP value represents the time difference from an upstream vessel to a downstream one. After the stent-placement procedure, there was a generalized decrease in TTP, BAT, rTTP, FWHM, WO, and AUC compared with the measurements on the prestent angiograms. Figure 5 shows the means of the BAT and TTP before and after stent treatment. From the map, we can see that the treatment reduced both the BAT and TTP in the neck and intracranial vascular segments.

\section{DISCUSSION}

Quantitative DSA facilitates real-time hemodynamic monitoring and helps determine the optimal angioplasty in carotid stenosis to avoid hyperperfusion ${ }^{4}$ and the end point of embolization in carotid cavernous fistulas. ${ }^{8,10}$ A previous study by Lin et al ${ }^{8}$ in 2012 showed significant reduction in rTTP at the ICA (in both PA and lateral views), A1, M1, and M2 after stent placement compared with pretreatment data. Lin et $\mathrm{al}^{10}$ reported a study of cerebral circulation time by calculating the rTTP relative to the cervical ICA on the PA view (same location as ICA 1 in our report) and relative to the cavernous segment of the ICA on the lateral view (same location as $\mathrm{ICA}_{2}$ in our report). We found that the poststenting mean circulation times of M1, M2, and PV (corresponding to rTTP of the following segments: $\mathrm{ICA}_{1}-\mathrm{M} 1, \mathrm{ICA}_{2}-\mathrm{M} 2$, and $\left.\mathrm{ICA}_{2}-\mathrm{PV}\right)$ in our study $(0.49,0.50$, and 4.14 seconds, respectively) were close to those of healthy controls $(0.46,0.58$, and $4.38 \mathrm{sec}-$ onds, respectively). ${ }^{10}$ Therefore, the cerebral circulation time returned to normal for M1, M2, and the PV after treatment. If the posttreatment cerebral circulation time does not decrease to normal, we have to consider a hemodynamically significant residual stenosis, arterial spasm, hidden tandem stenotic lesion, and microemboli. Those above-mentioned phenomena will alert medical professionals to do further clinical management.

Lin et $\mathrm{al}^{10}$ also evaluated the maximal slope of the wash-in phase (the same as WI in our study) in the time-concentration curve and found significant changes in the maximal slope before and after treatment for M2, SSS, and M1. Our study found that the WI had no significant peritherapeutic change for all ROIs evaluated (Table 1). Both previous studies and ours evaluated FWHM. The study of Lin et al showed significant change at M1 and M2 only. ${ }^{10}$ Our study showed significant changes for all arterial ROIs except A2. The discrepancy in maximal slope (WI in our 
Table 3: Results for the hemodynamic parameters with significant changes $(P<.05)$ after the stenting procedure in the lateral view

\begin{tabular}{|c|c|c|c|}
\hline ROI & $\begin{array}{l}\text { Before Treatment } \\
\text { (Mean } \pm S D)\end{array}$ & $\begin{array}{l}\text { After Treatment } \\
\text { (Mean } \pm S D \text { ) }\end{array}$ & No. \\
\hline \multicolumn{4}{|l|}{ TTP (sec) } \\
\hline $\mathrm{ICA}_{1}$ & $2.96 \pm 0.69$ & $2.47 \pm 0.25$ & 34 \\
\hline $\mathrm{ICA}_{2}$ & $3.30 \pm 0.81$ & $2.66 \pm 0.27$ & 34 \\
\hline$M 2^{2}$ & $3.98 \pm 0.98$ & $3.15 \pm 0.30$ & 34 \\
\hline $\mathrm{A} 2$ & $3.70 \pm 0.88$ & $2.97 \pm 0.41$ & 17 \\
\hline PV & $8.01 \pm 1.28$ & $6.79 \pm 0.68$ & 30 \\
\hline \multicolumn{4}{|l|}{ rTTP (sec) } \\
\hline$I C A_{1}-I C A_{2}$ & $0.34 \pm 0.24$ & $0.19 \pm 0.13$ & 34 \\
\hline $\mathrm{ICA}_{2}-\mathrm{M} 2$ & $0.68 \pm 0.25$ & $0.50 \pm 0.16$ & 34 \\
\hline $\mathrm{ICA}_{2}-\mathrm{A} 2$ & $0.66 \pm 0.38$ & $0.34 \pm 0.17$ & 17 \\
\hline$I \mathrm{ICA}_{2}-\mathrm{PV}$ & $4.73 \pm 1.00$ & $4.14 \pm 0.69$ & 30 \\
\hline M2-PV & $4.06 \pm 0.93$ & $3.67 \pm 0.65$ & 30 \\
\hline \multicolumn{4}{|l|}{ BAT (sec) } \\
\hline $\mathrm{ICA}_{1}$ & $1.39 \pm 0.37$ & $1.19 \pm 0.26$ & 34 \\
\hline $\mathrm{ICA}_{2}$ & $1.70 \pm 0.37$ & $1.33 \pm 0.29$ & 34 \\
\hline$M 2^{2}$ & $2.14 \pm 0.54$ & $1.65 \pm 0.34$ & 34 \\
\hline $\mathrm{A} 2$ & $2.17 \pm 0.69$ & $1.74 \pm 0.37$ & 17 \\
\hline PV & $4.88 \pm 0.94$ & $3.87 \pm 0.69$ & 30 \\
\hline SSS & $5.35 \pm 1.53$ & $4.39 \pm 1.09$ & 34 \\
\hline \multicolumn{4}{|l|}{ FWHM (sec) } \\
\hline $\mathrm{ICA}_{1}$ & $2.66 \pm 1.01$ & $1.93 \pm 0.41$ & 34 \\
\hline $\mathrm{ICA}_{2}$ & $2.66 \pm 0.93$ & $2.01 \pm 0.50$ & 34 \\
\hline $\mathrm{M} 2$ & $3.22 \pm 0.88$ & $2.54 \pm 0.65$ & 34 \\
\hline $\mathrm{A} 2$ & $2.90 \pm 0.84$ & $2.27 \pm 0.83$ & 17 \\
\hline \multicolumn{4}{|l|}{$\mathrm{WO}(\mathrm{AU} / \mathrm{s})$} \\
\hline $\mathrm{ICA}_{1}$ & $-217 \pm 110$ & $-281 \pm 91$ & 34 \\
\hline$I A_{2}$ & $-229 \pm 91$ & $-305 \pm 107$ & 34 \\
\hline$M 2^{2}$ & $-90 \pm 49$ & $-120 \pm 46$ & 34 \\
\hline $\mathrm{A} 2$ & $-79 \pm 37$ & $-116 \pm 34$ & 17 \\
\hline \multicolumn{4}{|l|}{$\mathrm{AUC}(\mathrm{AU} / \mathrm{sec})$} \\
\hline $\mathrm{ICA}_{1}$ & $1413 \pm 692$ & $1002 \pm 340$ & 34 \\
\hline $\mathrm{ICA}_{2}$ & $1561 \pm 753$ & $1160 \pm 385$ & 34 \\
\hline$M 2^{2}$ & $879 \pm 377$ & $750 \pm 299$ & 34 \\
\hline \multicolumn{4}{|l|}{$M E(A U)$} \\
\hline$A 2$ & $203 \pm 74$ & $234 \pm 72$ & 17 \\
\hline
\end{tabular}

Note:-No. indicates number of cases measured; $A U$, arbitrary unit.

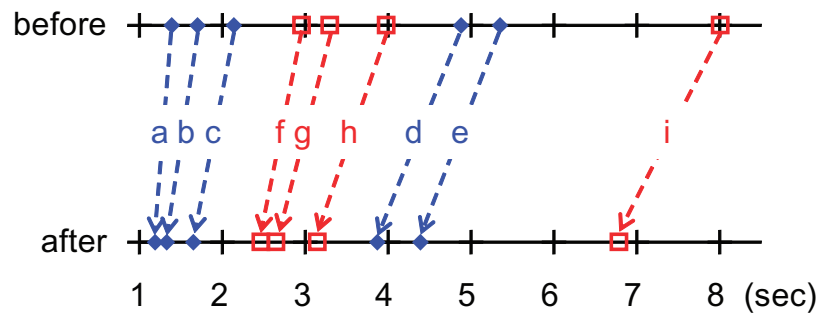

FIG 5. Comparison of the mean BAT (blue, a-e) and mean TTP (red, $\mathrm{f}-\mathrm{i})$ before (upper line) and after (lower line) treatment for different vascular ROIs in the lateral view: ICA $(a$ and $f$ ), ICA $(b$ and g), $M 2$ ( $c$ and h), PV (d and i), and SSS (e). The TTP and rTTP are reduced after treatment in the extracranial ( $a$ and $f$ ) and intracranial segments, being more obvious in segments between the ICA 1 and the PV ( $a-d$ and $f-i)$ and less obvious from PV to SSS ( $d$ and e).

study) and FWHM is most likely caused by the difference in calculation algorithm. Curve fitting in our study made the timeconcentration curve smooth; thus, the measured slope may be less than the maxillary slope obtained without curve fitting in the previous study. ${ }^{10}$

We evaluated the BAT in intracranial vascular points. The BAT was first used to make a color-coded image from angiograms obtained by 1 contrast media injection. ${ }^{6}$ The BAT has not been further evaluated for hemodynamic information of DSA, to our knowledge. Measurements of TTP, rTTP, ME, FWHM, WO, and AUC in venous structures were limited by the absence of late washout phase images. The calculation of BAT does not need the presence of a peak in the time-concentration curve and is not limited by late contrast media washout or early halting of the imaging process. Therefore, the BAT can be used in the evaluation of hemodynamic changes of veins with late washout, such as in conditions of dural sinus stenosis and dural sinus thrombosis.

During the DSA imaging, the contrast medium was injected at the common carotid artery proximal to the stenosis. The amount of contrast media injected for angiography before and after stent placement was the same, and it took longer for the contrast media to travel through the stenotic segment before stent placement. As a result, the TTP, which represents the contrast transit time, is significantly longer at the cervical ICA, M1, M2, A1, A2, and PV before stent placement (Tables 2 and 3). After the stenosis was relieved by stent placement, the transit time of contrast media through the previously stenotic segment recovered, and the washout process was quicker, reflected by a shorter TTP (Tables 2 and 3 ).

The carotid stenosis before stent placement not only caused a longer transit time in the stenotic segment that caused TTP prolongation at the $\mathrm{ICA}_{1}$ but also caused prolongation of the transit time in the distal segments of this artery, as shown by the significantly longer rTTP (Tables 2 and 3 and Fig 5). Prolongation of the transit time in the distal segments of this artery indicates that the blood flow rate was slower distal to the stenosis before stent placement. This finding is compatible with previous transcranial Doppler sonography demonstrations of reduced peak systolic velocity in the middle cerebral artery on the side of significant internal carotid artery stenosis. ${ }^{17}$

In this experiment, the sampling rate for acquiring DSA images was 6 frames/s. As a result, the temporal resolution of timeconcentration curves was $\Delta \mathrm{t}=0.17$ seconds. The theoretic $\mathrm{SD}^{18}$ of this digitized data in measuring BAT is $\Delta t / \sqrt{12}=0.05 \mathrm{sec}$ onds, which is relatively small compared with the SD of the BAT in Tables 2 and 3. The effect of the sampling rate on TTP is more complicated because we applied curve fitting to the measured data. Computer simulation is needed to study this effect. ${ }^{15}$

\section{CONCLUSIONS}

We compared the changes in 8 hemodynamic parameters before and after stent placement treatment of carotid stenosis. We found that the stent-placement procedure significantly reduced TTP, BAT, and WO at all arterial ROIs and FWHM and AUC at some arterial ROIs. The evaluation of venous structures by using TTP, rTTP, FWHM, WI, WO, ME, and AUC was limited if either the ME fell at the last image or a washout phase in the time-concentration curve was deficient. More images must be obtained for the late washout veins to evaluate venous ROIs with these 7 parameters.

The BAT showed significant changes not only at all arterial ROIs but also at the PV and SSS. Thus, the BAT can be used for the evaluation of cerebral circulation of venous structures when the TTP cannot be measured because of an incomplete time-concen- 
tration curve. This study also found that carotid stenosis resulted in longer transit times not only in the stenotic segment but also in the distal intracranial segments.

\section{REFERENCES}

1. Tenjin H, Asakura F, Nakahara $\mathrm{Y}$, et al. Evaluation of intraaneurysmal blood velocity by time-density curve analysis and digital subtraction angiography. AJNR Am J Neuroradiol 1998;19:1303-07 Medline

2. Hung SC, Liang ML, Lin CF, et al. New grading of moyamoya disease using color-coded parametric quantitative digital subtraction angiography. J Chin Med Assoc 2014;77:437-42 CrossRef Medline

3. Gölitz P, Struffert T, Lücking H, et al. Parametric color coding of digital subtraction angiography in the evaluation of carotid cavernous fistulas. Clin Neuroradiol 2013;23:113-20 CrossRef Medline

4. Lin CJ, Chang FC, Tsai FY, et al. Stenotic transverse sinus predisposes to poststenting hyperperfusion syndrome as evidenced by quantitative analysis of peritherapeutic cerebral circulation time. AJNR Am J Neuroradiol 2014;35:1132-36 CrossRef Medline

5. Levitt MR, Morton RP, Haynor DR, et al. Angiographic perfusion imaging: real-time assessment of endovascular treatment for cerebral vasospasm. J Neuroimaging 2014;24:387-92 CrossRef Medline

6. Cover KS, Lagerwaard FJ, van den Berg R, et al. Color intensity projection of digitally subtracted angiography for the visualization of brain arteriovenous malformations. Neurosurgery 2007;60:511-14; discussion 514-15 Medline

7. Strother CM, Bender F, Deuerling-Zheng Y, et al. Parametric color coding of digital subtraction angiography. AJNR Am J Neuroradiol 2010;31:919-24 CrossRef Medline

8. Lin CJ, Hung SC, Guo WY, et al. Monitoring peri-therapeutic cerebral circulation time: a feasibility study using color-coded quantitative DSA in patients with steno-occlusive arterial disease. AJNR Am J Neuroradiol 2012;33:1685-90 CrossRef Medline
9. Lin CJ, Luo CB, Hung SC, et al. Application of color-coded digital subtraction angiography in treatment of indirect carotid-cavernous fistulas: initial experience. J Chin Med Assoc 2013;76:218-24 CrossRef Medline

10. Lin CJ, Chang FC, Guo WY, et al. Changes of time-attenuation curve blood flow parameters in patients with and without carotid stenosis. AJNR Am J Neuroradiol 2015;36:1176-81 CrossRef Medline

11. Zhang Q, Xu R, Sun Q, et al. Exploring the value of using colorcoded quantitative DSA evaluation on bilateral common carotid arteries in predicting the reliability of intra-ascending aorta flat detector CT-CBV maps. AJNR Am J Neuroradiol 2015;36:960-66 CrossRef Medline

12. Thompson HK Jr, Starmer CF, Whalen RE, et al. Indicator transit time considered as a gamma variate. Cir Res 1964;14:502-15 CrossRef Medline

13. Coleman TF, Li Y. On the convergence of interior-reflective Newton methods for nonlinear minimization subject to bounds. Math Prog 1994;67:189-224 CrossRef

14. Coleman TF, Li Y. An interior trust region approach for nonlinear minimization subject to bounds. SIAM J Optim 1996;6:418-45 CrossRef

15. Bahn MM. A single-step method for estimation of local cerebral blood volume from susceptibility contrast MRI images. Magn Reson Med 1995;33:309-17 CrossRef Medline

16. Kenney JF, Keeping ES. Mathematics of Statistics: Part 1. 3rd ed. Princeton: Van Nostrand; 1962

17. Can U, Furie KL, Suwanwela N, et al. Transcranial Doppler ultrasound criteria for hemodynamically significant internal carotid artery stenosis based on residual lumen diameter calculated from en bloc endarterectomy specimens. Stroke 1997;28:1966-71 CrossRef Medline

18. Gray RM, Neuhoff DL. Quantization. IEEE Trans Inf Theory 1998;44: 2325-83 CrossRef 